\title{
3-D Natural Convection-Radiation Interactions in a Cube Filled with Gas-Soot Mixtures
}

\author{
TORU FUSEGI
}

Institute of Computational Fluid Dynamics

1-22-3 Haramachi, Meguro, Tokyo 152, Japan

\author{
BAKHTIER FAROUK \\ Department of Mechanical Engineering and Mechanics \\ Drexel University \\ Philadelphia, Pennsylvania 19104, USA
}

\section{KUNIO KUWAHARA}

Institute of Space and Astronautical Science

Sagamihara, Kanagawa 229, Japan

\section{ABSTRACT}

A high-resolution, 3-D finite-difference numerical study is performed on interactions of natural convection and surface/gas/soot radiation in a differentially heated cubical enclosure, which may be regarded as a simplified model for compartment fires. A robust gas/soot radiation model used in the analysis is based on the $P_{1}$-differential approximation method and the weighted sum of gray gas model. The 3-D characteristics of the thermal and flow fields are examined in detail by the state-of-the-art 3-D numerical visualization techniques. The effects of each mode of radiation are described. Overall, radiation is found to enhance the three-dimensionalities of the fields.

KEYWORDS : compartment fire modeling; natural convection-radiation interactions; gas-soot radiation; finite difference method

\section{NOMENCLATURE}

Bo $=$ Boltzmann number, $p_{0} c_{p_{0}} u_{0} / \sigma T_{\mathrm{o}}{ }^{3}$

$c_{p}=$ specific heat at constant pressure

$\mathrm{Fr}=$ Froude number, $u_{\mathrm{o}}^{2} / g_{0} L_{\mathrm{o}}$

$g=$ gravitational acceleration

$J=$ irradiance, $J=\int_{\Omega=4 \pi} I d \Omega$, where $I$ is the radiative intensity

$k=$ thermal conductivity

$L_{\mathrm{o}}=$ reference length (enclosure height)

$p=$ pressure

$p_{0}=$ reference pressure (hydrostatic pressure)

$\operatorname{Pr}=$ Prandtl number, $c_{p_{0}} \mu_{0} / k_{0}$

$\mathrm{Ra}=$ Rayleigh number, $g_{0} \beta_{0} c_{p \circ} \rho_{\mathrm{o}}^{2} L_{\mathrm{o}}^{3}\left(T_{H}\right.$ $\left.-T_{C}\right) / \mu_{0} k_{0}$

$$
\begin{aligned}
& \operatorname{Re}=\text { Reynolds number, } \rho_{\mathrm{o}} u_{\mathrm{o}} L_{\mathrm{o}} / \mu_{\mathrm{o}} \\
& t=\text { time } \\
& T=\text { temperature } \\
& T_{\mathrm{o}}=\text { reference temperature, }\left(T_{C}+T_{H}\right) / 2 \\
& T_{C}, T_{H}=\text { cooled and heated side wall } \\
& \quad \text { temper-atures } \\
& \begin{aligned}
u_{\mathrm{o}}= & \text { reference velocity, } \quad\left[g _ { \mathrm { o } } \beta _ { \mathrm { o } } L _ { \mathrm { o } } \left(T_{H}-\right.\right. \\
& \left.\left.T_{C}\right)\right]^{1 / 2}
\end{aligned} \\
& \begin{aligned}
u, v, w \quad \text { velocity components in the } x, y \\
\text { and } z \text { directions, respectively }
\end{aligned} \\
& \begin{array}{c}
W_{m}=\text { weighting function for the } m \text { th compo- } \\
\text { nent gray gas }
\end{array} \\
& x, y, z=\text { Cartesian coordinates }
\end{aligned}
$$


Greek symbols

$\beta=$ thermal expansion coefficient

$\delta=$ overheat ratio, $\left(T_{H}-T_{C}\right) / T_{\mathrm{o}}$

$\varepsilon_{w}=$ surface emissivity

$\kappa=$ absorption coefficient

$\mu=$ viscosity

$\rho=$ density

$\sigma=$ Stefan-Boltzmann constant $\tau=$ optical thickness, $\kappa L$

\author{
Subscripts \\ $m=m$ th component gray gas \\ $0 \quad=$ reference quantities (dimensional)
}

Superscript

$* \quad=$ dimensional quantities

\section{INTRODUCTION}

Interaction of natural convection (NC) and radiation (RD) is one of the crucial factors for the development of fires in a compartment. Despite the importance of the analysis on NC-RD interactions in enclosures, relatively little research has been reported in the literature due to the complicated nature of the problem [1 - 6]. The analysis must account for the strong coupling of the three modes of heat transfer (conduction, convection and radiation). Multi-dimensional radiation exchanges involve among surfaces, gases and soot. The non-grayness of gas radiation further complicates the analysis; due to this phenomenon, the integration of the radiative intensity over the entire wavelength is necessary in addition to the spatial integration over the whole solid angles, in order to evaluate the energy flux of radiatively participating gases. Hence, the exact treatment of NC-RD interactions is an extremely tedious and time-consuming process. Radiation models should be applied to solution of this class of problems [7]. A compromise between the accuracy and the efficiency may be found by considering the spherical harmonics $\left(P_{N}\right)$ approximation method and the weighted sum of gray gas (WSGG) model. The $P_{1}$ method with the WSGG model were employed in an analysis of turbulent forced flowradiation interactions in an industrial furnace filled with combustion gas mixtures [8]. The same procedure was later applied to a NC-RD interaction problem in a $\mathrm{CO}_{2}$-filled differentially heated cavity $[5,6]$.

The present paper investigates numerically NC-RD interactions in a differentially heated cubical enclosure of dimension of $L_{0}$, which is filled with gas-soot mixtures. The flow configuration of the problem is shown schematically in Figure 1 . The two vertical side-walls are maintained at uniform temperatures of $T_{C}\left(\right.$ at $\left.x^{*}=0\right)$ and $T_{H}\left(\right.$ at $x^{*}=L_{\mathrm{o}}$ ), where $T_{H}>T_{C}$. The remaining four walls are thermally insulated and all the interior surfaces are black for radiation. The medium is mixtures of carbon dioxide gas, water vapor and soot. The present problem may be regarded as a simplified model of NC-RD interactions in compartment fires.

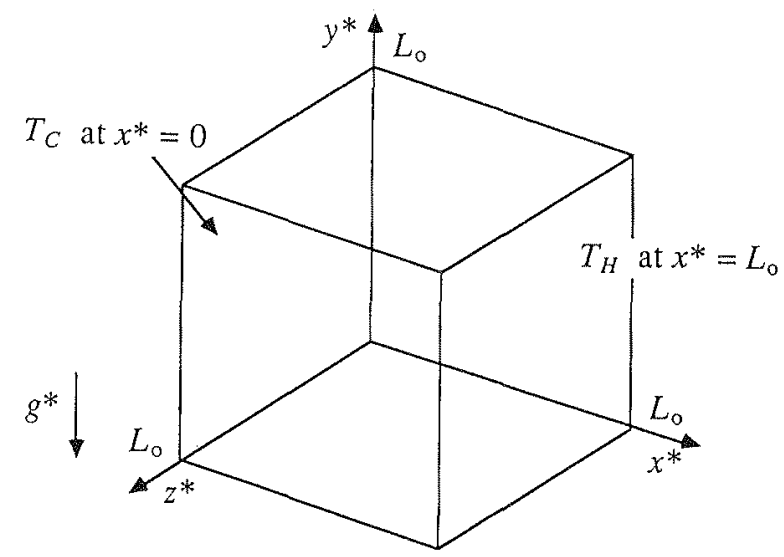

Figure 1 The flow geometry and the boundary conditions. The walls are radiatively black. They are also thermally insulated, unless otherwise indicated. 
The present numerical study is part of a long-term project for compartment fire simulations by a field method. Of particular interest of the present paper is a test of the robust radiation model for NC-RD interaction analysis in a 3-D enclosure. The past investigations on NC-RD interactions in compartment fires with gas-soot mixtures are limited to 2-D cases [11, 12]. With the advent of supercomputers, high-resolution, full 3-D compartment fire simulations may now be feasible. In extending the use of 2-D NC-RD interaction models in enclosures [1 - 6] to 3-D compartment fire situations, the applicability of those models should be demonstrated by a welldefined problem in enclosures. The present numerical exercise is performed as a direct extension of the prior 3-D NC-RD interaction analysis for carbon dioxide gas [6] and the 2-D computations with gas-soot mixtures [12] to cases of a differentially-heated, gas-soot mixturefilled 3-D enclosure.

\section{MATHEMATICAL MODEL}

\section{Governing Equations}

The governing equations consist of the Navier-Stokes equations, the energy equation and the transfer equation for the radiative intensity. The last equation is transformed to a system of differential equations for the irradiance of the component gray gases by using the $P_{1}$ approximation method and the WSGG model. The non-dimensionalized governing equations can be expressed in tensor form as:

$$
\begin{aligned}
& \frac{\partial \rho}{\partial t}+\frac{\partial}{\partial x_{j}}\left(\rho u_{j}\right)=0 \\
& \frac{\partial}{\partial t}\left(\rho u_{i}\right)+\frac{\partial}{\partial x_{j}}\left(\rho u_{j} u_{i}\right)=-\delta_{i 2} \frac{1}{\operatorname{Fr}}(\rho-1)-\frac{\partial p}{\partial x_{i}}+\frac{1}{\operatorname{Re}} \frac{\partial}{\partial x_{j}}\left(\frac{\partial u_{i}}{\partial x_{j}}+\frac{\partial u_{j}}{\partial x_{i}}-\delta_{i j} \frac{2}{3} \frac{\partial u_{k}}{\partial x_{k}}\right) \\
& \frac{\partial}{\partial t}(\rho T)+\frac{\partial}{\partial x_{j}}\left(\rho u_{j} T\right)=\frac{1}{\operatorname{Re} \operatorname{Pr}} \frac{\partial^{2} T}{\partial x_{j} \partial x_{j}}+\frac{1}{3 \mathrm{Bo}_{m}} \sum_{m=1}^{N} \frac{1}{\tau_{m}} \frac{\partial^{2} J_{m}}{\partial x_{j} \partial x_{j}} \\
& \frac{\partial^{2} J_{m}}{\partial x_{j} \partial x_{j}}+3 \tau_{m}^{2}\left(4 W_{m} T^{4}-J_{m}\right)=0, \quad m=1,2, \ldots, N
\end{aligned}
$$

where $\delta_{i j}$ is the Kronecker delta $\left(\delta_{i j}=1\right.$ if $i=j$, and $\delta_{i j}=0$ otherwise) and $N$ is the number of component gray gases. The fluid properties are assumed to be invariant except for the density. In order to study flows at a large overheat ratio, density variation is accounted for in all the terms of the governing equations. The density is computed from the ideal gas law.

The physical variables are non-dimensionalized in the following manner: $(x, y, z)=\left(x^{*}\right.$, $\left.y^{*}, z^{*}\right) / L_{0},(u, v, w)=\left(u^{*}, v^{*}, w^{*}\right) / u_{\mathrm{o}}, t=t^{*} u_{0} / L_{0}, p=\left(p^{*}-p_{0}\right) / \rho_{0} u_{0}^{2}, \rho=\rho^{*} / \rho_{0}, \mu=$ $\mu^{*} / \mu_{0}, k=k^{*} / k_{0}, g=g^{*} / g_{0}, T=T^{*} / T_{0}, J=J^{*} / \sigma T_{0}{ }^{4}$. A convective velocity, $\left(g_{\mathrm{o}} \beta_{\mathrm{o}} L_{\mathrm{o}} \Delta T_{\mathrm{o}}\right)^{1 / 2}$, is chosen as the reference velocity, $u_{\mathrm{o}}$. The reference temperature, $T_{\mathrm{o}}$, is set at the film temperature, $\left(T_{C}+T_{H}\right) / 2$.

\section{Non-Gray Gas Radiation Model}

The WSGG model postulates that the absorptivity of non-gray gases (and gas-soot mixtures) can be approximated by the sum of component gray gas absorptivities weighted with a temperature dependent factor. In the present analysis, three component gray gases are considered to represent the emissivity of the non-gray gas (mixture of carbon dioxide, water vapor and air). Two components are employed for the soot by following Smith et al. [9, 10]. In case of the gas-soot mixtures, eight equations (i.e., $N=8$ ) are required for irradiance, whose absorption coefficients are determined from the combination of absorption coefficients of the component gray gases. When soot is not considered, $N$ is reduced to three. The absorption coefficient of the gas-soot mixture, $\kappa_{m i x}$, is computed as $\kappa_{m i x}\left(T^{*}\right)=\Sigma^{3}{ }_{m=0} \Sigma^{2}{ }_{n=1}\left(P \kappa_{m, g a s}+\right.$ $\left.\kappa_{n, \text { soot }}\right) \cdot W_{m, g a s} \cdot W_{n, \text { soot }}$ with $\kappa_{n, \text { soot }}=f_{v} C_{n}, W_{m, g a s}=\Sigma^{4}{ }_{k=1} a_{k} T^{* k-1}$ and $W_{n \text {,soot }}=\Sigma^{4}{ }_{k=1} b_{k}$ $T^{* k-1}$, where $P$ is the partial pressure, $f_{v}$ is the volume fraction of soot and $C_{n}$ is the soot coefficient. The coefficients of WSGG model are taken from Smith et al. $[9,10]$. 
Boundary Conditions

In accordance with the problem description, the boundary conditions are:

$u=v=w=0$

$T=(2-\delta) / 2$ at $x=0, T=(2+\delta) / 2$ at $x=1$, and $\frac{\partial T}{\partial n}=\frac{\operatorname{Re} \operatorname{Pr}}{\mathrm{Bo}}\left( \pm q_{s}-\sum_{m=1}^{N} \frac{1}{3 \tau_{m}} \frac{\partial J_{m}}{\partial n}\right)(6)$

at $y=0$ and $z=0$ (with the positive sign), and at $y=1$ and $z=1$ (with the negative sign)

$\frac{\partial J_{m}}{\partial n}= \pm 3 \tau_{m} \frac{\varepsilon_{w}}{2\left(2-\varepsilon_{w}\right)}\left(J_{m}-4 W_{m} T^{4}\right), \quad m=1,2, \ldots, N$

at $x=0, y=0$ and $z=0$ (with the positive sign), and at $x=1, y=1$ and $z=1$ (with the negative sign) where $n$ denotes the coordinate normal to the surface and $q_{s}$ is the surface radiative flux, which is computed as $q_{s}=W_{0} \Sigma_{i} F_{1-i}\left(T_{w}{ }^{4}-T_{i}{ }^{4}\right)$, where $F_{1-i}$ is the shape factor for radiation between surface elements 1 and $i$. The summation is taken for all the surfaces that the element 1 can see. For the gas mixtures, $W_{0}$ is defined as $W_{0}=1-\Sigma_{j=1}^{3} W_{j}$.

\section{SOLUTION METHOD}

The governing equation system (1) - (7) are discretized by a control-volume based finite difference procedure. Numerical solutions are obtained by an iterative method, together with the pressure correction algorithm, SIMPLE [13]. The present study employs the Strongly Implicit Scheme [14] to accelerate convergence characteristics of solutions. SIP is applied to the planes of constant $z$ in order to simultaneously determine dependent variables in the $x$ and $y$ directions on each plane.

The convection terms in the momentum equation (2) are approximated by the QUICK scheme modified for non-uniform grids [15], while those in the energy equation (3) are discretized by a hybrid scheme [13]. The QUICK scheme involves a third-order accurate upwind differencing, which possesses the stability of the first-order upwind formula and is free from substantial numerical diffusion experienced with the usual first-order schemes.

The entire enclosure is considered as the computational domain. The number of grid points for computations is $41 \times 41 \times 41$. Variable grid spacing is used to resolve steep gradients of the velocity and the temperature near the walls. Care is taken to distribute several grid points inside the boundary layer formed near the walls.

In order to calculate the surface radiative flux, the shape factor for an elemental surface with respect to other elemental surfaces needs to be evaluated. This is done by computing the shape factors between a control-volume surface of the finite difference mesh system located on the boundary and any other control-volume surfaces that it can see. Simpson's formula for numerical integration is employed for multiple integration of the formula for the shape factor between two differential elements taken on the control-volume surfaces, from which the desired shape factors between two control-volume surfaces are computed.

\section{RESULTS AND DISCUSSION}

Computations are carried out at $R a=4.50 \times 10^{6}$ and for cases of carbon dioxide gas, the mixture of carbon dioxide and water vapor, and the gas $\left(\mathrm{CO}_{2}\right.$ and $\left.\mathrm{H}_{2} \mathrm{O}\right)$-soot mixture. In order to isolate the effects of each radiation mode (surface, gas and gas-soot radiation), several sets of calculations are made by selectively neglecting one or more of the above modes. In all the computations, the reference pressure of the mixture is $1 \mathrm{~atm}$. For the gas-soot mixture, the partial pressure of the $\mathrm{CO}_{2}-\mathrm{H}_{2} \mathrm{O}$ mixture and the volume fraction of soot are, respectively, 0.25 atm and $10^{-7}$. They are typical values for combustion of hydrocarbon fuels with moderate soot generation [10]. The partial pressures of $\mathrm{CO}_{2}$ and $\mathrm{H}_{2} \mathrm{O}$ are equal in the mixture. The temperature difference of the vertical side walls is maintained at $555 \mathrm{~K}$. The same value is specified for the reference temperature, $T_{0}$; hence, the overheat ratio, $\delta$, is equal to unity. Note 
that both $T_{\mathrm{o}}$ and $\delta$ must be given in order to correctly evaluate the effects of radiation in the present problem. In order to compare results at the same $R a$, the enclosure height, $L_{0}$, is modified depending on the mixtures: the difference in the fluid properties is adjusted by changing $L_{0}$. Height is set equal to $0.0762 \mathrm{~m}$ for the case of $\mathrm{CO}_{2}$, while $L_{\mathrm{o}}=0.110 \mathrm{~m}$ is specified when the $\mathrm{CO}_{2}-\mathrm{H}_{2} \mathrm{O}$ mixture as well as the gas-soot mixture are considered.

The computations were carried out on the HITACHI S820 supercomputer at ICFD. The system has a maximum computational speed of 3 GFLOPS. The computer code was vectorized. The typical CPU time for obtaining converged solutions was approximately 30 minutes per run for the natural convection cases. However, the CPU time is increased to approximately 3 hours in the radiation cases. About $200 \mathrm{MB}$ of memory space was used for a complete computation. First, results for the natural convection mode (without radiation) and the surface/gas radiation mode for carbon dioxide gas are inspected. The perspective views of the thermal and flow fields are illustrated in Figure 2. The isothermal surfaces and the magnitude of the vorticity are shown for the natural convection and surface/gas radiation modes. The fields in the half domain, $0.5<z<1$, are seen to be the mirror images of the others $(0<z<0.5)$.

If radiation is neglected (i.e., natural convection only), the temperature and flow field patterns exhibit the centro-symmetry with respect to the line of $x=0.5$ and $y=0.5$. Slight deviations from the exact centro-symmetry are attributed to density variation effects, which are significant under the large temperature difference specified in the present study. In the natural convection mode, the fields can be characterized by the presence of thermal and hydrodynamic boundary layers near the vertical side walls $(x=0$ and 1$)$ and the almost-stagnant inner core region. The three-dimensionalities (i.e., $z$-variations) of the fields are confined into areas close to the end walls ( $z=0$ and 1 ).

When surface/gas radiation is included, dramatic changes take place. The centro-symmetry disappears due to an increase in the temperature of the thermally insulated plates (the horizontal and vertical end-walls) near the heated isothermal side wall $(x=1)$. The overall temperature of the fluid attains higher values than the natural convection case through radiative exchanges, which involve among surfaces and gas volumes. In the figure, this is evident by downward shift of the isotemperature surface for $T=1$ (the average temperature). The intensity of the flow also increases due to a significant increase in areas of high temperature surfaces, compare the increments of the isovorticity surfaces. Large values of the vorticity are still concentrated in regions near the walls; however, these areas are spread extensively over the walls, indicating uniform flows which are similar to those observed in conduction-dominated natural convection are present in the flow field. The thermal boundary layers formed in the vicinity of the isothermal side-walls ( $x=0$ and 1 ) become thicker. The $z$-variations of the thermal fields are appreciable; the isotemperature surfaces fold inward adjacent to the end walls $(z=0$ and 1$)$.

In order to scrutinize the three-dimensionalities of the fields, projected velocity vectors in constant $x$ and $y$ planes are depicted in Figures 3 and 4, respectively. The reference velocity scale is indicated for each case. The results for the natural convection and surface/gas radiation for $\mathrm{CO}_{2}$ are presented side-by-side for comparison, as was attempted in Figure 2.

The combined effects of the vertical $(v-)$ and transverse $(w-)$ velocities are illustrated in Figure 3, which shows the cross-sectional patterns of the flow field in the planes parallel to the isothermal side-walls $(x=0$ and 1$)$. All the results exhibit the exact symmetry with respect to the mid $z$-plane $(z=0.5)$. A large portion of the flow displayed at the plane of $x=0.1$ is located within the boundary layer near the cooled wall $(x=0)$, which develops in the negative $y$ direction. For the natural convection case, the cold-wall boundary-layer flow is seen to be discharged into the almost-stagnant central core near the bottom plate $(y=0)$. In the mid $x$ plane $(x=0.5)$, weak secondary vortices are visible near the edges. The flow patterns at $x=$ 0.25 and 0.75 are closely antisymmetric with respect to the line of $y=0.5$ and $z=0.5$ in the natural convection mode. The breakdown from the exact antisymmetry is due to the effect of variable density, as previously remarked. In all the cases, significant $z$-variations are noticeable in the velocity vector field.

In the surface/gas radiation mode, the flow field patterns differ considerably at each $x$ plane. Overall, the flow intensity is seen to increase, compare the velocity vector scales attached to each case. The magnitudes of the fluid motion in the interior core region are appreciable and the flow patterns become uniform. The discharging of the boundary layer flows into the core, 

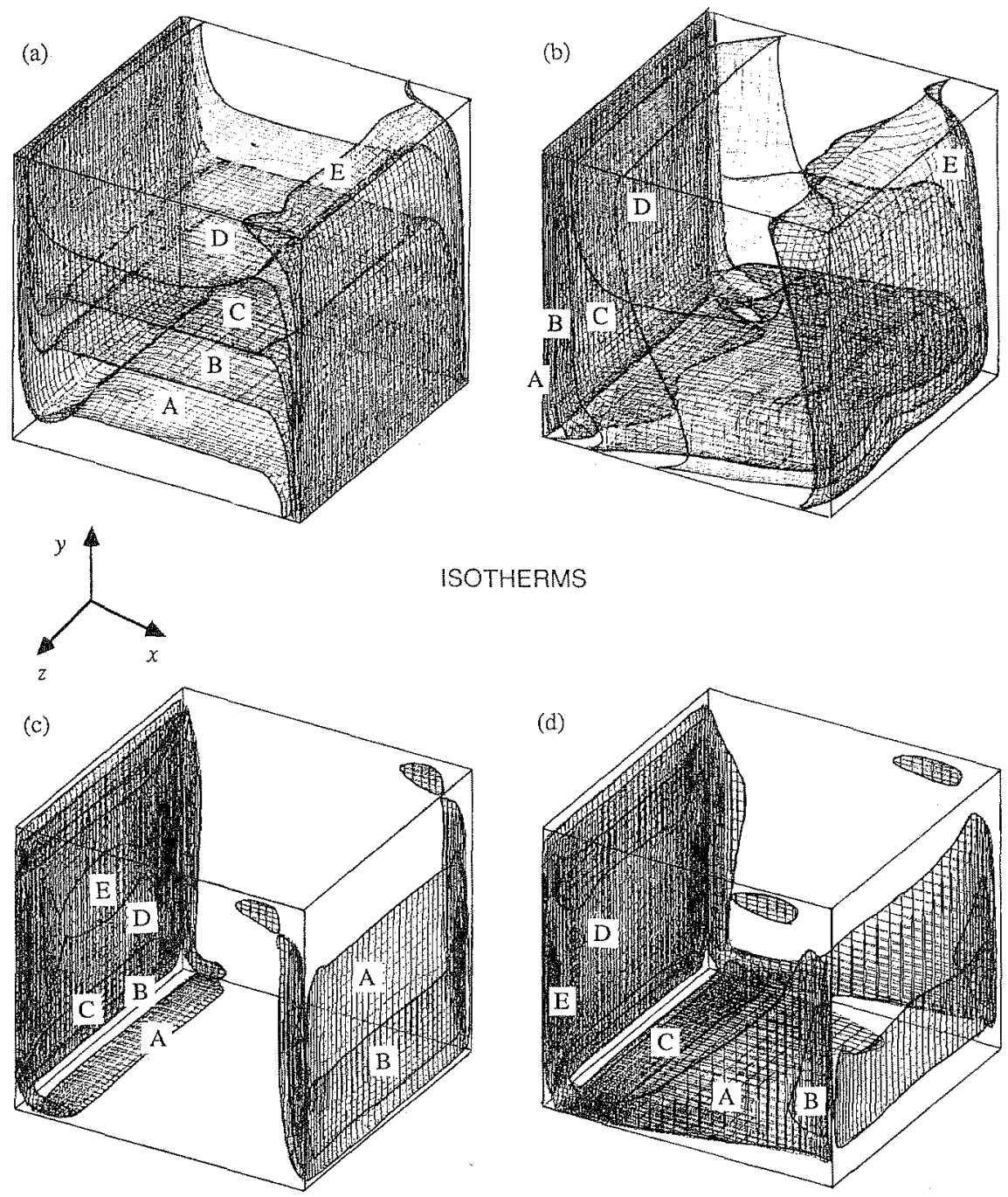

ISOTHERMS

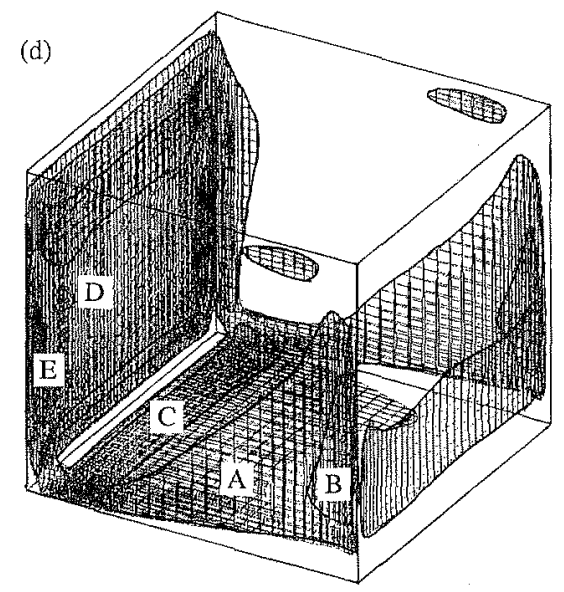

ISOVORTICITY SURFACES

Figure 2 Perspective views of the surfaces of isotemperatures and isovorticities (absolute values) [contour levels: (a) \& (b) $0.6667(\mathrm{~A}), 0.8333(\mathrm{~B}), 1.0(\mathrm{C}), 1.167(\mathrm{D}), 1.333(\mathrm{E})$; (c) 15 (A), 30 (B), 45 (C), 60 (D), 75 (E); (d) 18 (A), 36 (B), 54 (C), 72 (D), 90 (E)] 
NATURAL CONVECTION
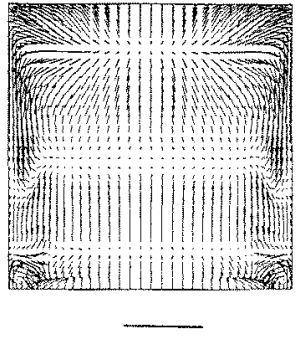

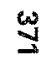
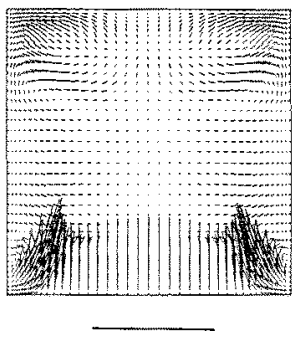

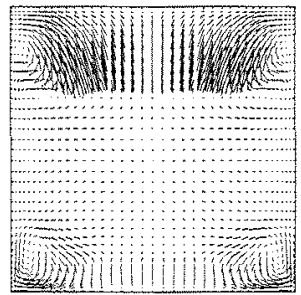

$4 / 10$

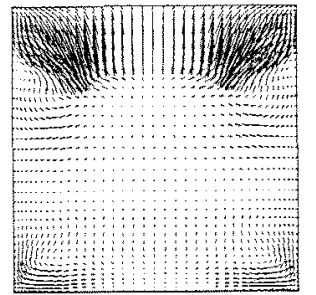

$\longrightarrow$

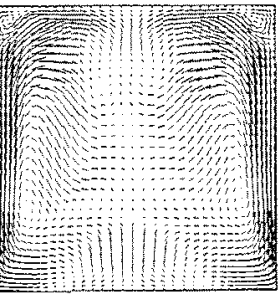

$-$

$x=0.75$

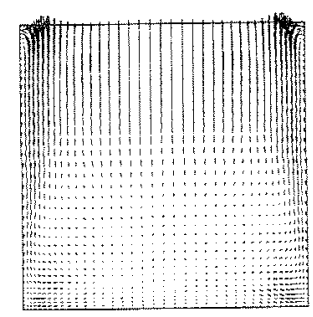

$-$

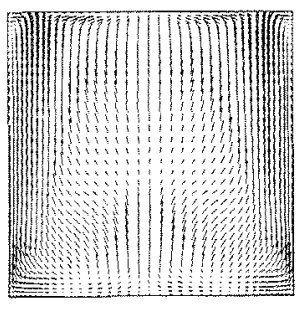

$x=0.9$

Figure 3 Projected velocity vectors on planes of $x=$ constant. The scaie shows the magnitude of the reference velocity. 
NATURAL CONVECTION

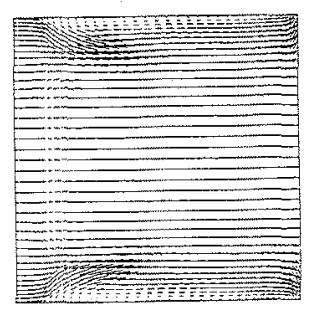

$-$

$\stackrel{w}{N}$

SURFACE/GAS RADIATION

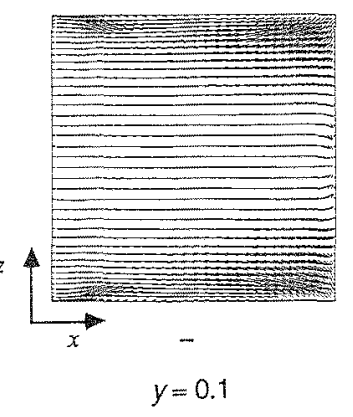

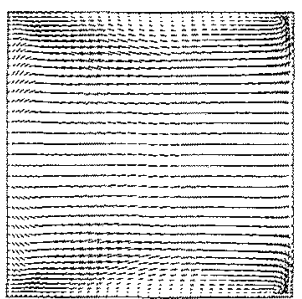

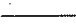

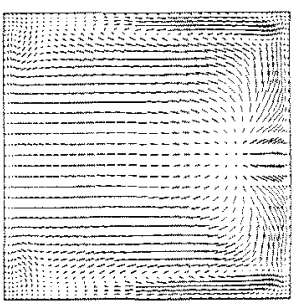

$y=0.25$

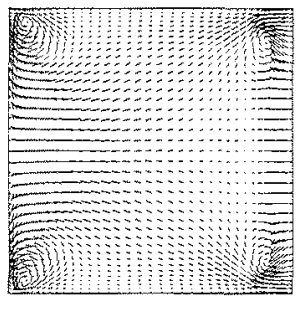

$u_{0} / 10$

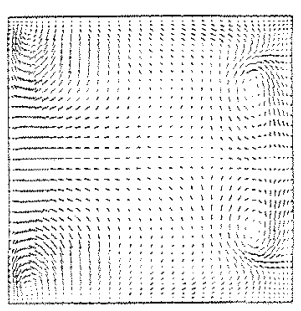

-

$y=0.5$

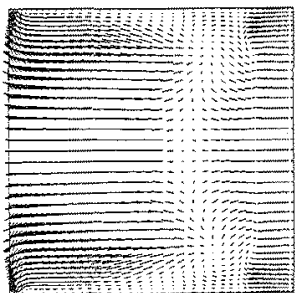

-

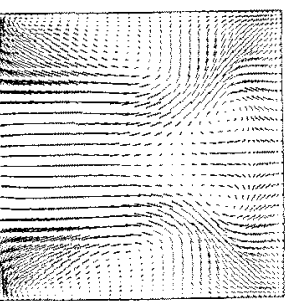

$y=0.75$

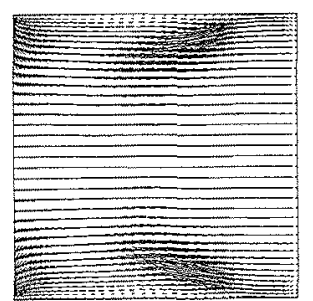

$-$

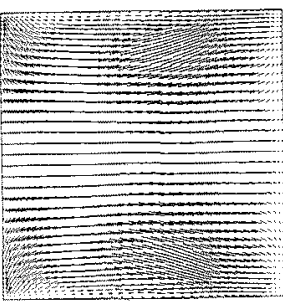

$y=0.9$

Figure 4 Projected velocity vectors on planes of $y=$ constant. The scale shows the magnitude of the reference velocity. 
as clearly seen in the natural convection case, is not apparent in the surface/gas radiation mode. Three-dimensionalities are notable in these cross-sectional fields.

The velocity-vector patterns at different elevations $(y=$ constant) are shown in Figure 4 . In both modes of radiative transfer (no radiation, i.e., natural convection, and surface/gas radiation), the results at $y=0.1$ and 0.9 illustrate the flows directed from the cooled wall $(x=$ 0 ) toward the heated wall $(x=1)$, and vice versa. In the natural convection case, the fields are nearly symmetric with respect to the mid $y$-plane $(y=0.5)$, compare the vector plotting for $y=$ 0.1 with that of $y=0.9$, and the result for $y=0.25$ with that of $y=0.75$. As a strong contrast, this is not at all found in the surface/gas radiation mode. An interesting flow pattern is noted in $y=0.25$ in this mode of radiation. A multi-layer flow structure is observable, which consists of thin layers flowing in the positive $x$-direction near the end walls $(z=0$ and 1$)$, and the dominant layer in the center with its flow direction opposite to the secondary layers. The generation of such multi-layer flows is associated with the strong bending of the constant temperature surfaces in the vicinity of the thermally insulated end-walls, see also Figure 2 . In the $y=0.5$ plane, weak secondary-vortices appear near the edges in both natural convection and surface/gas radiation modes. The vortices close to the heated plate move toward the symmetry plane $(z=0.5)$ when radiation is included. Overall, three-dimensionalities of the flow fields are more pronounced with radiation. Qualitatively similar trends are observed in changes due to radiation for the cases of the mixture $\left(\mathrm{CO}_{2}-\mathrm{H}_{2} \mathrm{O}\right)$ and the soot-mixture.

Effects of each radiation modes (surface, gas and gas/soot radiation) are studied by the average Nusselt number at the isothermal walls, which is presented in Table 1 . Note that the results obtained from the two values of the enclosure length, $L_{0}$, (and, consequently, the Boltzmann number - the interaction parameter for convection and surface radiation -) are combined in the table. This is due to the fact that, in the present calculations, the Rayleigh number is held fixed at $4.5 \times 10^{6}$ for all the cases considered $\left(\mathrm{CO}_{2}\right.$ and $\mathrm{CO}_{2}-\mathrm{H}_{2} \mathrm{O}$ mixtures). The Prandtl number of the two fluids is approximately the same, i.e., 0.68 , at the reference temperature, $T_{0}$. The first three cases, namely, NC, SR and SGR(CO2) constitute a set, in which the results for SR and NC are generated in the computations by turning off gas radiation and surface/gas radiation calculations, respectively. The enclosure length, $L_{0}$, is equal to $0.0762 \mathrm{~m}$ in this data set. When radiation is present, i.e., SR and SGR(CO2), the heat transfer rate to the isothermal side-walls is increased to approximately four times of the $\mathrm{NC}$ case. A slight reduction in the Nusselt number is observed for SGR(CO2) compared to the SR data, due to absorption of energy by the radiatively participating gas.

Table 1 The average Nusselt number at the isothermal walls $\left(\mathrm{Ba}=4.5 \times 10^{6}\right)$

\begin{tabular}{|c|c|c|c|c|}
\hline $\mathrm{NC}^{a_{1}}$ & $\mathrm{SR}^{b, 1}$ & SGR(CO2) $)^{C_{1}}$ & SGR(MIX) ${ }^{d} 2$ & $\mathrm{GStR}^{\theta, 2}$ \\
\hline 15.2 & 63.6 & 62.0 & 72.3 & 80.5 \\
\hline
\end{tabular}

Notes: a) natural convection; $b$ ) surface radiation; ${ }^{c)}$ surface/gas radiation for carbon dioxide gas; ${ }^{d}$ surface/gas radiation for the carbon dioxide and water vapor mixture; ${ }^{\theta)}$ soot/gas radiation for the $\mathrm{CO}_{2}$ $\mathrm{H}_{2} \mathrm{O}$-soot mixture; ${ }^{1)} L_{0}=0.0762 \mathrm{~m}, \mathrm{BO}=90.5$ (for radiation cases only); ${ }^{2)} L_{0}=0.110 \mathrm{~m}, \mathrm{BO}=71.4$.

The last two cases, namely, SGR(MIX) and GStR, form another set, for which the enclosure length of $0.11 \mathrm{~m}$ is considered. Due to a decrease in the Boltzmann number, the surface radiation mode at this $B o$ would attain a higher value than the SR case at $B o=90.5$. Inclusion of soot is found to increase the heat transfer rate. It should be realized that in all the previous results for radiation [SR, SGR(CO2), and SGR(MIX)], surface radiation effects through the optical windows of the gases are accounted for; however, by assuming a gray medium for the gas-soot mixture, surface radiation is not taken into account explicitely in the calculations.

\section{CONCLUSIONS}

Interactions of NC-RD in a 3-D differentially heated cubical enclosure are investigated numerically for gas-soot mixtures. In the thermal boundary condition used in the present study, radiation is found to enhance the three-dimensionalities of the fields. The thermally insulated surfaces (the horizontal and vertical end-walls) reach at high temperatures, which generates large magnitudes of fluid motion in the $z$-direction near these walls. An increase in the overall 
temperature of the insulated plates is mainly attributed to surface radiation effects when soot is absent. For gas/soot radiation case, the computed field patterns are similar to those in the surface/gas radiation mode. The gas-soot mixture is assumed to be gray in the model used for the present investigation.

Effects of each radiation mode (surface, gas and gas/soot radiation) are illustrated in the present computations. When a relatively large overheat ratio is specified, as $\delta=1$ in the present case, surface radiation through non-gray gases is considered to play the dominant role for the heat transfer enhancement. Gas radiation modifies slightly the heat transfer rate by absorbing energy by the participating gases.

The applicability of the robust radiation model, which is based on the $P_{1}$-approximation method and the WSGG model, to a 3-D differentially heated cubical enclosure filled with gassoot mixtures is demonstrated by the present study. In order to extend this procedure to realistic 3-D compartment fire simulations, local variations of mixtures as well as soot must be handled in an appropriate manner, which are generated by the presence of openings in a room. One of the other important factors not addressed in the present analysis is effects of the high Rayleigh number, which is usually in the turbulent flow range due to the size of the enclosure. Steadystate characteristics are studied in the present computations. From the viewpoint of fire safety in comparmtnet, transient problems should be considered. The proper treatment of convection and radiation need much care due to mismatch in time scales for these transport phenomena. Studies on these effects are now under way.

\section{REFERENCES}

1. Chang, L. C., Yang, K. T. and Lloyd, J. R., "Radiation-Natural Convection Interaction in TwoDimensional Complex Enclosures", Journal of Heat Transfer, 105, 89 - 95, 1983.

2. Zhong, Z. Y., Yang, K. T. and Lloyd, J. R., "Variable-Property Natural Convection in a Tilted Enclosure with Thermal Radiation", in Numerical Methods in Heat Transfer, Vol. III, pp. 195 - 214, John Wiley, New York, 1985 .

3. Fusegi, T. and Farouk, B., "Radiation-Convection Interactions of a Non-Gray Gas in a Square Enclosure", in Heat Transfer in Fire, ASME-HTD Vol. 73, pp. 63-68, 1987.

4. Yucel, A., Acharya, S. and Williams, M. L., "Combined Natural Convection and Radiation in a Square Enclosure", Proc. 1988 Natl. Heat Transfer Conf., ASME HTD-96, Vol. 1, pp. $209=218,1988$.

5. Fusegi, T. and Farouk, B., "Laminar and Turbulent Natural Convection-Radiation Interactions in a Square Enclosure Filled with a Nongray Gas", Numerical Heat Transfer, part A, 15, $303-322,1989$.

6. Fusegi, T., Ishii, K., Farouk, B. and Kuwahara, K., "Three-Dimensional Natural Convection-Radiation Interactions in a Cubical Enclosure Filled with a Nongray Gas", Numerical Heat Transfer, part A, 1991 (in press).

7. Yang, K. T., "Numerical Modeling of Natural Convection-Radiation Interactions in Enclosures", Proc. 8th Int. Heat Transfer Conf., Vol. 1, pp. 131 - 140, 1986.

8. Song, T. H. and Viskanta, R., "Interaction of Radiation with Turbulence: Application to a Combustion System", Journal of Thermophysics and Heat Transfer, 1, 56 - 62, 1987.

9. Smith, T. F., Shen, Z. F. and Friedman, J. N., "Evaluation of Coefficients for the Weighted Sum of Gray Gases Model", Journal of Heat Transfer, 104, 602 - 608, 1982.

10. Smith, T. F., Al-Turki, A. M., Byun, K.-H. and Kim, T. K., "Radiative and Conductive Transfer for a Gas/Soot Mixture between Diffuse Parallel Plates", Journal of Thermophysics and Heat Transfer, 1, 50 . $55,1987$.

11. Lloyd, J. R., Yang, K. T. and Liu, Y. K., "A Numerical Study of One Dimensional Surface, Gas and Soot Radiation for Turbulent Buoyant Flows in Enclosures", Proc. 1st Nat. Conf. on Numerical Methods in Heat Transfer, pp. 142 - 161, 1979.

12. Fusegi, T. and Farouk, B., "Numerical Study of Interactions of Turbulent Convection and Radiation in Compartment Fires", Fire Science and Technology, 8, 15 - 28, 1988.

13. Patankar, S. V., Numerical Heat Transfer and Fluid Flow, chapter 6, Hemisphere, Washington, DC, 1980.

14. Stone, H. L., "Iterative Solution of Implicit Approximation of Multi-Dimensional Partial Differential Equations", Journal of Numerical Analysis, 5, 530 - 558, 1968.

15. Freitas, C. J., Street, R. L., Findikakis, A. N. and Koseff, J. R., "Numerical Simulation of ThreeDimensional Flow in a Cavity", International Journal for Numerical Methods in Fluids, 5, 561 - 575, 1985.

16. Fusegi, T., Hyun, J. M., Kuwahara, K. and Farouk, B., "A Numerical Study of 3-D Natural Convection in a Differentially Heated Cubical Enclosure", International Journal of Heat and Mass Transfer (in press, 1991). 